\title{
Vibroacoustic Therapy: Sound Vibrations in Medicine
}

\author{
Chris Boyd-Brewer, MA. FAMI
}

\begin{abstract}
Vibroacoustic therapy is a recently recognized technology that uses sound in the audible range to produce mechanical vibrations that are applied directly to the body. The technology uses speakers or transducers placed within mats, mattresses, chairs, recliners, tables, or soft furniture to provide a physiologic and auditory experience. Benefits include pain management, anxiety relief, symptom reduction, physical therapy, and health improvement.
\end{abstract}

Three general designs of vibroacoustic technology have been developed over the past 30 years, with unique capabilities and purposes. The designs differ in the type of vibration-generating systems used; the frequencies selected for stimulus; the extent to which sound vibrations are processed, measured and monitored' and the qualities and patterns of resonance in the vibrating surfaces.

Interest in incorporating vibroacoustics in patient care practices may be attributable to the nonpharmacologic and noninvasive nature of the technology. Because some models of vibroacoustic technology can be administered easily in nursing care, this may have also led to broad use of vibroacoustics in medical and wellness practices.

Research and/or development of vibroacoustic use include applications to manage pain; reduced symptoms for patients in chemotherapy; reduce stress; distract patients during biopsies, aspirations, and other procedures; increase range of motion (ROM) and muscle tone and in physical therapy or rehabilitative therapy following knee replacement; help prepare patients for surgery or those who are recovering; and provide sensory stimulation for people who are hearing impaired and developmentally disabled.

Vibroacoustics can also increase quality of life and be used to manage behavior in psychiatric settings, geriatric facilities, child life centers, and palliative care facilities.

\section{Background and History}

Vibrations in the audible range have been used to help bring patients back to mental, physical and emotional balance in many cultures, 1,2 Current medical technology utilized vibration in varying degrees of intensity and frequency. Ultrasonic frequencies, above the audible range, assist with diagnoses via ultrasound technology. Very high-frequency vibrations are used to shatter kidney stones and to treat tissue contractures. Complementary health practitioners use forms of inaudible sound below $20 \mathrm{~Hz}$ for infrasonic vibratory treatment, such as sound waves used by chiropractors to relieve joint and muscle pain.

Vibroacoustic equipment was developed between 1970 and the late 1980 s when innovative individuals recognized the value of felt vibrations combined auditory effects of music or sound. Norwegian educator and therapist Olav Skille experimented with low-frequency sounds between 20 and $120 \mathrm{~Hz}$ to generate vibrations for children with severe physical and mental handicaps. He developed the first VibroAcoustic Chair (Skille, Levanger, Norway) in the late 1980s, using one pulsed, sinusoidal frequency as the sound source for generating vibrations blended with music for listening.4 In the 1970s, Petri Lehikoinen, a clinical psychologist at Helsinki University, Finland, created the physioacoustic method based on scanning the body with sinusoidal sound between 27 and $113 \mathrm{~Hz}$ and specially selected listening music.

In 1985, United States inventor Byron Eakin released his vibroacoustic system as a relaxation tool... Research indicates that these models may reduce anxiety, tension, and fatigue as well as managing pain and disease symptoms.

Kris Chesky, director of education and research of the Texas Center for Music and Medicine at the University of North Texas, Fort Worth, designed a vibroacoustic unit in the late 1980s to facilitate scientific study of vibroacoustics. His Music Vibration Table (MVT) targets frequencies within the effective range of a natural pain-suppressing mechanism that can be stimulated by vibration. 10 This sophisticated design allows for scientific measurement and control of the mechanical vibrations 
that make an impact on the person being stimulated. The MVT uses computers for complex sound processing and feedback capabilities.

\section{Key Acoustic and Vibration Characteristics}

Vibroacoustic technology is based on key acoustic principles. Sound frequencies that are input into vibroacoustic devices become mechanical vibrations felt by the body. A significant acoustic consideration in vibroacoustic design is how vibrations are received and experienced by patients. Important factors include the resonance characteristics of the vibrating surface, or vibrating membrane, and the spatial distribution of vibrations over the surface.11 Variation in how materials receive sound can make a significant difference in the vibration a patient receives.

Placement of speakers or transducers in the equipment and the resonant quality of the vibrating membrane affect the spatial distribution of vibrations across the surface. The surface area displaced by a person on the equipment and his or her weight can also affect spatial distribution. The pattern of vibration dispersion will determine which parts of the body receive vibration dispersion will determine which parts of the body receive vibration and to what degree this happens. Most designers have experimented with these elements and each model has different qualities and patterns of resonance.

The sound stimulus may use specific frequencies with or without music. Various sound properties can be manipulated to attain specific experiences. Sine waves have been historically considered to have unique health benefits in many cultures and are used in some vibroacoustic models. A sine wave, or pure tone, flows with a precisely matched increase and decrease of amplitude. Visually, sinusoidal waves have a smoothly rising and falling curvature exactly replicated with each cycle. These pure tones are used in some models because they do not produce overtones that could affect the vibration experience or alter the frequency dose.

Some vibroacoustic designs focus on the application of sound frequencies between 27 and $135 \mathrm{~Hz}$ and experimentation has indicated the resulting mechanical vibrations create strong physical responses in patients.4,5,13 Use of these frequencies with Skille's VibroAcoustic equipment and selective low-frequency (SLF) models using speakers is also necessary because of the way the ear amplifies frequencies above $135 \mathrm{~Hz}$ (note. - Olav Skille and Stephen Deuel at Inner Soulutions have been collaborating since 2003).

The range of 60-600 Hz has been emphasized in certain vibroacoustic applications because this frequency range is known to stimulate Pacinian corpuscles, which play a key function in pain perception.11,14-16 Most models (note - Such as the Tactile Sound Tables and Mats built by Inner Soulutions at Vibroacoustic.org) also incorporate full-frequency music at least for listening, based on benefits that have been recognized in music therapy,17,18 and to provide a more interesting experience for patients.

\section{Sound Vibration Processing}

Sound vibration-processing techniques are used in some vibroacoustic designs to create patterns of sound that affect the experience of felt vibrations. Pulsed sound originated from VibroAcoustic technology while the physioacoustic method developed pulsation, scanning and directionality. These techniques may be generated during treatment but may also be used as compositional techniques in prerecorded music.

Pulsed sound blends two relatively close frequencies, for example, $70 \mathrm{~Hz}$ and $70.5 \mathrm{~Hz}$. Together, they produce a fast-pulsed sound. Musicians would say the frequencies in this example are "out of tune" with on another.

Sound is most often pulsed in vibroacoustics at a slow rate of speed. It is believed that pulsed sounds create a more pleasant sensation for patients and add a relaxing element to treatment. 4 Research by Tony Wigram, associate professor at the Institute for Music and Music Therapy, Aalborg, Denmark, compared pulsed sound lengths of 6, 10 and 14 seconds, peak to peak, and a pure sinusoidal tone. Subjects in this study expressed personal preferences for more slowly pulsed sound waves but study data did not confirm that slower-pulsed sounds induced greater relaxation. 
Pulsation varies the volume of low-frequency sound within a certain time sequence. Based on the theory that continual vibration may cause numbness in the body, alternating periods of more and less intense vibration in pulsation are believed to alleviate stimulation fatigue, or overstimulation, and to increase treatment effectiveness. Experimentation with SLF designs indicated that prolonged stimulation may cause muscle contractions that can be avoided via pulsation and that pulsation can induce greater muscle relaxation.

Scanning was originally developed to increase muscle relaxation through the physioacoustic method. The theory behind scanning is that each muscle resonates to a specific and unique frequency. Frequencies are scanned above and below approximate known resonant frequencies of target muscle(s). Physioacoustic explanations indicate that approximate resonant frequencies have been determined in physiotherapy and research at Stockholm's Karolinska Institute in Sweden has supported this principle. 5 Within the scanned frequency spectrum the target muscle(s) will, at some time, resonate with the frequency with which it has an innate resonance and receive benefit from this stimulation. Scanning is designed so that the desired frequency will be stimulated dozens of times in a single vibroacoustic session. Each time the muscle responds, greater relaxation is believed to occur.13 This theory is based on the physics principle of sympathetic resonance and is believed to work with body tissues as well.

The principle of directionality refers to movement of sound vibrations from one speaker or transducer to another. The movement is felt as a wave of vibration up or down the body and can enhance the vibroacoustic experience.

\section{Music in Vibroacoustics}

While specific frequencies and vibration processing techniques have proven to be effective in vibroacoustics, music may also hold essential value. Research has shown that live and recorded music can be used in treating illness and maintaining health.

Chesky, Michel, and Kondraske presented the Two-Pronged Approach conceptual model of vibroacoustics, highlighting the powerful synergy created from integration of physiologic sound vibration and psychological stimulation from music listening.11 Vibration and music together may be more powerful than either element independently and may be especially useful in certain instances.

An important aspect of music in vibroacoustics may be the broad range of frequencies within music. The effects achieved with SLF techniques may occur naturally in the wider range of frequencies in music. There are also potential benefits or problems from the overtone frequencies within music as opposed to the pure sine waves used in some methods.

Most vibroacoustic technologies use music either in addition to low frequencies for listening benefit or as the primary stimulus. A patient's enjoyment of music may play a key role in motivating the use of vibroacoustics. The majority of patients are interested in using vibroacoustics because it is a treatment modality that is pleasing and comforting, unlike many invasive and potentially unpleasant medical procedures.

Recorded music has been designed specifically to enhance vibroacoustic effects and some commercial recordings also work well. The MVT has the ability to process frequencies within any music selection, thus offering unlimited music selection options and allowing patients to choose their own music for effective treatment.

\section{Vibroacoustic Technology}

Three basic vibroacoustic systems have evolved. I have categorized these as full frequency music (FFM), selective low frequency (SLF), and quantified mechanical vibration (QMV) systems (note the Tactile Sound Tables and Mats fit into both the FFM and SLF).

FFM systems have demonstrated effectiveness for initiating relaxation, reducing anxiety, and assisting in pain management. FFMs use multiple speakers or transducers to project musical vibrations to the body from a single source. Generally, a standard sound system is used, although FFM equipment has the potential to be driven through a computer or other electronic instrument. 
Other than the standard amplifier adjustments used in commercial sound systems, felt vibrations are not processed, monitored, or measured in FFM designs and doses cannot be determined.

Music has been composed for FFM systems to maximize vibration effects and qualities via specific low frequencies, pulsed sound, pulsation, scanning, and directional sound movement. FFM units play any recordings although vibroacoustic effects may be limited with commercial music. FFM models are capable of responding to a wide range of frequencies as well as overtones produced by the music.

FFM models are the lease expensive systems and, because they are easy to use, offer a wide range of applications in medical and wellness facilities. These unite provide multiple benefits derived from relaxation but are limited in specific treatment capabilities.

SLF systems are designed to provide relaxation and treat pain and disorders with vibrations generated by selected low-frequency sounds. Both Skille's VibroAcoustic device and Lehikoinen's physioacoustic models are SLF systems. These designs focus on sinusoidal sound waves within a range of 20 to $135 \mathrm{~Hz}$. Frequencies are computer-generated during treatment or are prerecorded.

Processed vibrations are administered with or without accompanying music. When music is used, it is generally slow, harmonious, and relaxing. SLF unites use pulsed sound waves or pulsation, scanning, and directionality.

Some SLF systems are capable of measuring vibration parameters at the sound sources but no models have been developed that measure or monitor doses received at the vibratory surfaces. Skill and therapists who worked with his methods gathered a large amount of data on SLFs effectiveness for use in reducing tension, physical therapy, pain management, and treatment of various disorders.4 Some contraindications have been experienced I the use of low frequencies,5,13 which have not been noted in FFM and QMV designs. The SLF systems are not currently available in the United States but a new version of the physioacoustic device is being released in early 2004.*

The most complex technology, QMV, represented by Chesky's Music Vibration Table10,21 is intended to be used to treat pain and other disorders. The MVT is uniquely designed to ensure that measurable and repeatable vibratory doses are delivered to patients. Quantification of the soundstimulated mechanical vibration dose is made at the vibrating surface.

The MVT has three important features: (1) is the ability to quantify and monitor vibration parameters at the delivery point rather than the source point (vibration measurement parameters are based upon National and International Standards for HumanVibration11). (2) The technology allows for controlling frequency characteristics and for selected frequencies to be equalized and profiled. This minimized the chance of exposing a patient to one or more frequencies at a higher level than others and provides for measurement and application of frequency-specific vibration doses especially at 60 to $600 \mathrm{~Hz} .11$ (3) Th system has improved membrane resonance and more even distribution of vibration across the vibrating surface, increasing dosage accuracy. More research of the QMV concept is necessary to develop it for medical use.

\section{Vibroacoustic Applications in Medicine}

\section{Relaxation}

Relaxation and resultant anxiety reduction have been continuously noted as significant outcomes of vibroacoustic therapy by technology designers and researchers. At the 1994 National Association of Music Therapy conference in Los Angeles, California, George Patrick, Ph.D., chief of Recreation Therapy at the National Institutes of Health (NIH), presented the theory that vibroacoustic therapy triggers the relaxation response 6 and that this state is responsible for many stress-mediated medical and nursing care benefits of vibroacoustics.

Dr. Patrick developed a relaxation program in 1992 at the NIH Clinical Center that included Somatron FFM vibroacoustic therapy. This program has since been used to treat more than 15,000 patients. + Dr. Patrick gathered data on vibroacoustic treatment effects from 267 patients with a wide variety of medical conditions including cancer; heart, lung and blood disorders; infectious diseases; and mood disorders. Vibroacoustic therapy improved patients' perceived levels or relaxation by 33 percent and here was a 54-percent combined reduction of patient-perceived 
intensity of symptoms of pain, tension, fatigue, nausea, headache, and depression.

Additional research has yielded similar results. Nurses in the chemotherapy unit of Jupiter Medical Center, Jupiter, Florida, reported reduction of anxiety by 62.8 percent in 41 vibroacoustic sessions with 27 patients who received chemotherapy for cancer.7 Fatigue was reduced by 61.6 percent.

In a study with 33 patients who underwent knee replacement or revision, and who received SLF physioacoustic treatment during presurgery preparations, there was a 21-percent decrease in tension.23 Patients with Rett syndrome responded to SLF vibroacoustics with increased relaxation, reduction of anxiety, and reduction of hyperventilation and syndrome-related behaviours.

Positive affective responses produced by vibroacoustic treatment have been noted in patients with physiologic disorders.27,28 In a study at the Clinic of Psychiatry in Helsinki, Finland, researchers used physioacoustic treatment successfully to reduce anxiety, tension, and psychosomatic pains.

\section{Physical Therapy}

Vibroacoustics has a long history of experimentation in physical therapy. Treatment benefits include reduction of muscle tones, increased ROM, reduction of muscle spasticity, and sensory stimulation for patients with severe disabilities.

Skille began his research with children who were severely mentally and physically handicapped. By 2001, Skille and other therapists has gathered data from more than 40,000 hours of treatment. Many of the results found were related to benefits derived from reduced muscle tenstion.

Research on muscle spasticity on patients with cerebral palsy found that SLF treatment could reduced high muscle tone and accompanying movement restriction caused by increased rigidity and stiffness in these patients. ROM was also increased with treatmen and relaxation induced by vibroacoustic treatment resulted in reduced muscle spasms. SLF is also considered to be a viable treatment for fixed deformities for certain patients with cerebral palsy and may curb the development of fixed deformities. 3 People with Rett syndrome also benefit from relaxed muscle tone and experience improvement in muscle control with vibroacoustic treatment .

Vibroacoustics for increasing ROM has expanded into other medical areas. Research at Duke University Medical Center, Durham, North Carolina, using SLF Physioacoustic treatment for patients, over age 55, during physical therapy following total knee replacements showed greater passive ROM. Of 9 patients who were given the experimental treatment, 66 percent were able to achieve a 90 -degree bend compared to 44 percent of the 9 control subjects.

\section{Surgery and Other Medical Procedures}

Vibroacoustic research and experimentation in surgical units and procedures areas has recently been undertaken with SLF and FFM systems. A study with 33 patients who underwent knee replacements or revisions produced a 21-percent reduction of tension after patients received SLF physioacoustic treatment postsurgically. Subjects in the experimental group went home an average of one half-day earlier, used fewer mg of pain medication, and expressed more satisfaction with the care they received than did control group subjects.

Heart surgeons have used SLF physioacoustic therapy during cardiac surgery recovery. Ina study using SLF treatment, patients' use of sedative and pain medication decreased and average ventilator time was reduced from 17 hours to 7 hours. Time spent in the cardiac surgical unit decreased from averages of 36 hours to 18 hours and overall hospital stay was reduced to an average of 5 days from a 9-day average.

Vibroacoustics has reduced patients' anxiety during medical procedures. Vibrotactile and auditory music reduced agitation and physiologic instability following suctioning in infants with bronchiopulmonary dysplasia.32 Anecdotal reports from the Tampa Children's' Hospital at St. Joseph's, Tampa, Florida, from 1990 to 1997, using a specially designed vibroacoustic examination table for a various procedures noted that infants increased time spent in a quiet, alert state and had higher oxygen saturation levels.

Research at Dallas Children's' Hospital, Texas, is underway to determine the effectiveness of vibroacoustics for distraction during biopsies, aspirations, portable catheter maintenance, and other procedures. Nursing staff members have reported seeing lowered anxiety levels in patients during 
these procedures when vibroacoustic treatment was used.

Research is being conducted to compare vibroacoustics with other distraction mediums and to quantify the degree of anxiety reduction. Research is also ongoing at the Women's' Diagnostic Center in Jupiter, Florida, where FFM treatment is used before breast-cancer biopsies. Experimental variables include anxiety levels and amounts of Valium requested and/or used.

\section{Pain Management}

Vibroacoustic therapy has produced significant gains in pain relief and management. Dr. Patrick's NIH program evaluation produced a 63-percentreduction of perceived pain and he has suggested that this pain relief is relaxation-induced. 6 Patients in chemotherapy with FFM vibroacoustics have experienced 61-percent reductions of pain, probably via the same mechanism.

QMV technology was used for pain reduction with patients who have rheumatoid arthritis. Patients who listened to music while experiencing music vibration had a 64-percent reduction in pain while patients receiving only music had a24-percet reduction in pain and a placebo group experienced a 2-percent increase in pain.

Research has also been conducted with SLF technologies producing pain relief for 25 patients in a hospice. Patients who received vibroacoustic treatment used significantly less medication in 48 hours, experienced less discomfort, and reported lower pain intensity than did controls.

Twenty (20) women at Duke University Medical Center who had surgery for ovarian, endometrial, or cervical cancer experienced significant pain reduction with treatment.

The nonpharmacologic nature of vibroacoustics makes this treatment an important painmanagement tool. While it may not work in every instance, it is a promising complementary method that may reduced the need for pain medication and provide a pleasant alternative to medication. The ease of use and implementation of the FFM models makes vibroacoustics a promising nursing care practice.

\section{Additional Applications and Comments on Research}

Additional positive effects have been found by Wigram and others in extensive reviews of vibroacoustic research and cast study data, including the technique's ability to:

- Stimulate blood flow throughout the body making pressure sores and ulcers heal faster

- Reduce swelling caused by excess tissue fluid

- Ease sore ad aching muscles, alleviate high- and low-back pain, and alleviate stiffness in joints by improving joint motion 1

Anecdotal information indicates effects on pulmonary disorders including asthma, cystic fibrosis, pulmonary emphysema, and metachromatic leucodystrophy.

Some research to date has been derived from anecdotal evidence, case studies or program evaluations that used no control groups. In addition, not all research methods incorporated standards of vibration parameters and may have used equipment without capability for specifically measuring doses.11 Certainly, additional research is needed, especially studies that will use standardized measurements and appropriate statistical analyses.

Some studies indicate that vibroacoustics may not work for treating certain medical conditions or for all patients. While these studies have not been duplicated and changes in methods may provide effective treatment, it is worth paying attention to this research.

Patients with idiopathic Parkinson's disease experienced no improvement in disease symptoms but had moderate advancement in motor abilities and ability to perform tasks of daily living.41 Patients with fibromyalgia experienced no significant changes in perception of pain with vibroacoustics but were able to tolerate greater pressure on tender joints.

Anxiety levels in two learning disabled students were significantly different in one case study.43 A study that compared vibroacoustic treatment during physical therapy with music and movement- 
based physiotherapy showed that vibroacoustic treatment did not have a greater effect on ROM improvement.

Vibroacoustic therapy offers an important treatment option for many disorders, symptoms, and pain situations but there are factors that may make the treatment less effective in some instances or with certain individuals. A consideration is that vibroacoustics is passive and isolates patients during treatment. When interaction or activity are important, vibroacoustics may be less effective or useful as a secondary treatment.

\section{Conclusions}

Vibroacoustic technology is an effective treatment for relaxation, anxiety reduction, muscle-tension reduction, and pain management. Vibroacoustics has potential for treating disorders and for use during medial procedures. The method is effective for a wide variety of medical and wellness programs and has promise for others. The technology of vibroacoustics has evolved to provide greater understanding of how it works but continued development of technology and research on effective protocols and dosages is necessary to meet this technology's full potential.

\section{References}

1. Campbell, D. Music: Physician for Times to Come. London: Quest Books, 1991.

2. Pratt, R., Jones R. Music and medicine: A partnership in history. In J Arts Med, 1988; 6: 377-389.

3. Hooper J. An introduction to vibroacoustic therapy and an examination of its place in music therapy practice. Br J Music Ther 2001;5: 69-77.

4. Skille O. Manual of vibroacoustic therapy. Levanger, Norway: ISVA Publications, 1991. Online document at: quadrillo.tripod.com/ quadrillo/ndex-4.html.

5. Lehikoinen, Petri. The physioacoustic method. In: Wigram T, Dileo C, eds. Music Vibration and Health, Cherry Hill, NJ: Jeffrey Books, 1997; 209-216.

6 . Patrick $G$. The effects of vibroacoustic music on symptom reduction: inducing the relaxation response through good vibrations. IEE Eng Med Biol Mag 1999;18: 97-100.

7. Brewer C. Boyd, Coope V. Effectiveness of vibroacoustic music for pain and symptom management in outpatient chemotherapy treatment. First International Institute on Arts in Healing, May 16-17, 2003. Christine E. Lynn College of Nursing, Florida Atlantic University, Boynton Beach, FL

8. Walters $C$. The psychological and physiological effects of vibrotactile stimulation via a Somatron, on patients awaiting scheduled gynecological surgery. J Music Ther1996;33: 261287.

9. Brewer C. The Somatron Pain and Anxiety Management Program. The Somatron Corporation, 2000.

10. Chesky KS, Michel DE. The Music Vibration Table (MVT): Developing a technology and conceptual model for pain relief. Music Ther Perspect 1991;9: 32-37.

11. Chesky KS, Michel DE, \& Kondraske G. Developing methods and techniques for scientific and medical application of music vibration. In: Spintge R, Dron R eds. Music Medicine, vol 2. St. Louis, MO: MMB Music. 1996;2: 227-241.

12. Wigram, T. The effects of vibroacoustic therapy on clinical and non-clinical populations [doctoroal dissertation, St. George's Medical School, London University 1996]. Online document at: quadrillo.tripod.com/ quadrillo/index-4.html

13. Wigram T. The development of vibroacoustic therapy. In: Wigram T, Dileo C, eds. Music Vibration and Health, Cherry Hill, NJ: Jeffrey Books, 1997: 11-26.

14. Lundenberg T, Nordemarr R, Ottoson D. Pain alleviation by vibratory stimulation. Pain 1984;20: 2-44.

15. Quillian TA, Sato $M$. The distribution of myelin and nerve fibres from Pacinian Corpuscles. J Physiol, 1955;129: 167-176.

16. Hubbard SJ. A study of rapid mechanical events in a mechanoreceptor. J Physiol 1958; 141: 198-218.

17. Dileo C. The context of music and medicine. In: Wigram T, Dileo C, eds. Music Vibration and Health. Cherry Hill, NJ: Jeffrey Books, 1997: 37-48. 
18. Weldin C, Eagle C. An historical overview of music medicine, In: Applications of Music Medicine, Washington, DC: National Association for Music Therapy 1991: 7-27.

19. Wigram $T$. The effect of amplitude modulation of the pulsed sinusoidal low frequency tone used as a stimulus in Vibroacoustic (VA) therapy: In: Wigram T, Dileo C, eds, Music Vibration and Health. Cherry Hill, NJ: Jeffrey Books, 1997; 133-42.

20. Wigram T. The effect of VA therapy on multiply handicapped adults with high muscle tone and spasticity. In: Wigram T, Dileo C, eds. Music Vibration and Health, Cherry Hill, NJ: Jeffrey Books, 1997: 143-48.

21. Chesky KS. The effects of music and music vibration using the MVT ä on the relief of rheumatoid arthritis pain. [Dissertation] In: Abstracts International, 1992;53(8), 2725B. [University Microfilms No. AAC9300593].

22. Benson H, Klipper M. The Relaxation Response. New York: Avon Books, 1976.

23. Burke $M$, Phillips-Bute $B$, Parker-Vail t. Positive effects of music therapy and vibration on satisfaction in TKA patients. Alexandria, VA: Dept. of Veterans Affairs: Second Annual Leadership Conference: Pain Management and End of Life Care, 2001.

24. Cass H, Slonims V, Weekes L, Wigram T, Wisbeach A. Therapy services for Rett Syndrome: How well does provision match specific needs? Paper presented to the Royal Society of Medicine, London, 1995.

25. Wigram T, Cass, $\mathrm{H}$. The role of music therapy in a clinic for children and adults with Rett Syndrome. Paper presented to the BSMT Conference, London, 1995.

26. Wigram, T. Vibroacoustic therapy in the treatment of Rett Syndrome. In: Wigram T, Dileo C. (eds.) Music Vibration and Health, Cherry Hill, NJ: Jeffrey Books, 1997: 149-155.

27. Madsen CK, Standley JM, Gregory D. The effect of a vibrotactile device, Somatron, in physiological and psychological responses: musicians versus nonmusicians. J Music Ther 1991;28: 14-22.

28. Walters C. The psychological and physiological effects of vibrotactile stimulation via a Somatron, on patients awaiting scheduled gynecological surgery. J Music Ther 1996: 33: 261287.

29. Skille O, Wigram T, Weekes L. Vibroacoustic therapy: The therapeutic effect of low frequency sound on specific physical disorders and disabilities. J Br Music Ther 1989;3: 610. 30. Burke M, Thomas K. Use of physioacoustic therapy to reduce pain during physical therapy for total knee replacement patients over age 55, In: Wigram T, Dileo C, eds. Music Vibration and Health. Cherry Hill, NJ: Jeffrey Books, 1997: 99-106.

31. Butler C, Butler P. Physioacoustic therapy with cardiac surgery patients. In: Wigram T, Dileo C, eds. Music Vibration and Health. Cherry Hill NJ: Jeffrey Books, 1997: 197-204.

32. Burke M, Walsh J, Oehler J, Gingras JA.Music therapy following suction: Four case studies. Neonatal Network 1995: 14: 41-49.

33. Jones, L. Vibroacoustics with hospitalized children. In: Wigram T, Dileo C, eds. Music Vibration and Health. Cherry Hill,

$\mathrm{NJ}$ : Jeffrey Books, 1997; 189-196. 34. Michel DE, Chesky K. Music and music vibration for pain relief: Standards in research. In: Spintge R, Dron R, eds. Music Medicine, vol 2. St. Louis: MMB Music, 1996: 218-226.

35. Burke, MA. Feasibility of Physioacoustic Therapy in Cancer Care. Report for NIH Grant \#1 R43 CA 75899-01. A1, 1999.

36. Burke M. Effects of physioacoustic intervention on pain management of postoperative gynecological patients. In: Wigram T, Dileo C, eds. Music Vibration and Health. Cherry Hill, $\mathrm{NH}$ : Jeffrey Books, 1997: 107-123.

37. Boakes M. Vibrtactile stimulaton [internal document]. London: British Association Occupational Therapists, 1990.

38. Skille O. Vibroacoustic therapy: Music therapy. Am Asoc Music Ther 1989;8; 61-77.

39. Vincente P, Manchola I, Serna E. The use of vibroacoustics in idiopathic Parkinsons disease. In: Wigram T, Dileo C, eds. Music Vibration and Health. Cherry Hill, NJ: Jeffrey Books, 1997; 125-131.

40. Chesky KS, Russell IJ, Lopez Y, Kondraske G. Fibromyalgia tender point pain: A doubleblind, placebo-controlled pilot study of music vibration using the Music Vibration Table. J Musculoskeletal Pain 1997; 5: 33-52. 
41. Hooper J, Lindsay B. The use of the Somatron in the treatment of anxiety problems with clients who have learning disabilities. In Wigram T, Dileo C, eds. Music Vibration and Health. Cherry Hill, NJ: Jeffrey Books, 1997; 169-176.

42. Wigram T. The effect of vibroacoustic therapy compared with music and movement based physiotherapy on multiply handicapped patients with high muscle tone and spasticity. In:

Wigram T, Dileo C, eds. Music Vibration and Health. Cherry Hill, NJ: Jeffrey Books, 1997, 6985.

Published In Alternative and Complementary Therapies, October 2003.

Notes added by Stephen Deuel - Inner Soulutions - Vibroacoustic.org 